This is the pre-peer reviewed version of the following article: Berger \& Escobar (2016, Int. Stat. Rev.), which will be published in final form at

http://onlinelibrary.wiley.com

This article may be used for non-commercial purposes in accordance with Wiley Terms and Conditions for Self-Archiving.

\title{
Variance estimation of imputed estimators of change for repeated rotating surveys
}

\author{
Yves G. Berger \\ Emilio L. Escobar \\ University of Southampton, UK. \\ Numerika, Mexico. \\ Y.G.Berger@soton.ac.uk \\ Emilio@numerika.mx
}

November 26, 2015

\begin{abstract}
A common problem in survey sampling is to compare two cross-sectional estimates for the same study variable taken on two different waves or occasions. These cross-sectional estimates often include imputed values to compensate for item non-response. The estimation of the sampling variance of the estimator of change is useful to judge whether the observed change is statistically significant. Estimating the variance of a change is not straightforward due to the rotation in repeated surveys and imputation. We propose using a multivariate linear regression approach and show how it can be used to accommodate the effect of rotation and imputation. The regression approach gives design-consistent estimation of the variance of change when the sampling fraction is small. We illustrate the proposed approach using random hot-deck imputation, although the proposed estimator can be implemented with other imputation techniques.
\end{abstract}

Key Words: Longitudinal surveys; Missing data; Nonresponse; Overlapping samples; Rotation; Unequal inclusion probabilities. 


\section{Introduction}

Measuring change over time is a central problem for many users of social, economic and demographic data. The primary interest of many users is often in changes or trends from one time period to another. Smith, Pont and Jones (2003) recognised that assessing change is one of the most important challenge in survey statistics. A common problem is to compare two cross-sectional estimates for the same study variable taken on two different waves or occasions. These cross-sectional estimates often include imputed values to compensate for item non-response (e.g. Lohr, 2009, ch. 8). The estimation of the variance of an estimator of change is useful to judge whether the observed change is statistically significant. Covariances play an important role in estimating the variance of an estimated change and they are not straightforward to estimate with repeated surveys due to rotation.

We propose to use a multivariate linear regression approach to estimate these covariances. The proposed estimator is not a model-based estimator, as it is valid even if the underlying model does not fit the data (Berger and Priam, 2010, 2016). We show how this approach can be used to accommodate the effect of imputation. The regression approach gives design-consistent estimation of the variance of change when the sampling fraction is small. We illustrate the proposed approach using random hot-deck imputation, although the proposed estimator can be implemented with any other imputation techniques.

\section{Rotating surveys}

The estimation of variance of change would be relatively straightforward if crosssectional estimates were based on the same sample. Furthermore, because of rotations 
that is used in repeated surveys, cross-sectional estimates are not independent. Let $s_{1}$ and $s_{2}$ denote respectively the first and the second wave samples. The samples $s_{1}$ and $s_{2}$ are usually not completely overlapping sets of units, because repeated surveys use rotation designs which consist in selecting new units $\left(k \in s_{2} \backslash s_{1}\right)$ to replace old units ( $k \in s_{1} \backslash s_{2}$ ) that have been in the survey for a specified number of waves. Without lost of generality, we assume that $s_{1}$ and $s_{2}$ have the same sample size $n$. Let $n_{12}$ denote the sample size of the common sample, $s_{12}=s_{1} \cap s_{2}$. The units sampled on $s_{12}$ represent usually a large fraction of $s_{1}$; that is, $n_{12} / n$ is usually large. We denote the overall sample by $\tilde{s}$ where $\tilde{s}=s_{1} \cup s_{2}$. The size of the overall sample is denoted by $\tilde{n}=\#(\tilde{s})$. The Figure 1 gives a visual representation of the samples considered.

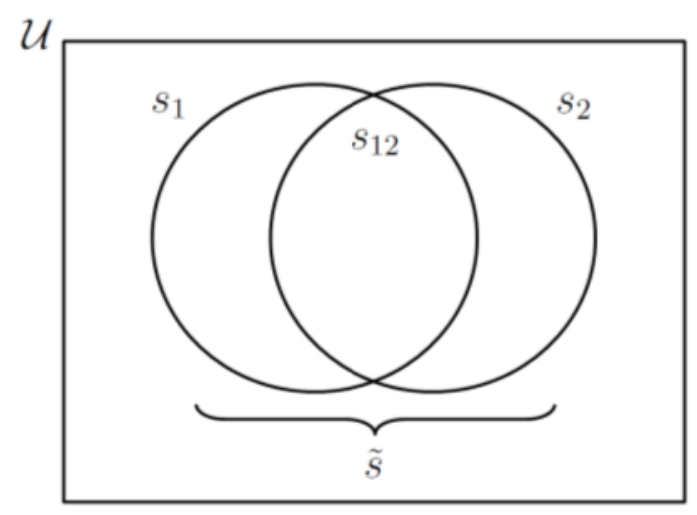

Figure 1: The overall sample $\tilde{s}=s_{1} \cup s_{2}$.

It is important to clarify that units in $s_{1} \backslash s_{2}$ are not available at the second wave, and units in $s_{2} \backslash s_{1}$ are not available at the first wave. This non-availability is not due to non-response (introduced in Section 3), but due to the rotation. The imputation is used to impute missing values due to non-response, not to impute the units which are not available because of the rotation. In other words, those units in $s_{2} \backslash s_{1}$ are not necessarily imputed at the first wave, and units in $s_{1} \backslash s_{2}$ are not necessarily imputed at the second wave. 
We assume that the rotation sampling design is such that $n$ and $n_{12}$ are fixed quantities. This class contains standard rotating sampling designs such as the rotating systematic sampling design (Holmes and Skinner, 2000), the rotation groups sampling design (e.g. Kalton, 2009; Gambino and Silva, 2009), the rotating design that was proposed by Tam (1984) and the permanent random numbers rotating design (e.g. Ohlsson, 1995; Nordberg, 2000). Rotating sampling designs are widely used in practice for labour force surveys (e.g. Holmes and Skinner, 2000). Rotation design is also used for the European Union Statistics on Income and Living Conditions (EU-SILC) social survey (Eurostat, 2012).

Let $y_{\ell ; k}$ denote the value of the variable of interest $y_{\ell}$ for the wave $(\ell=1,2)$. Suppose, we wish to estimate the absolute change

$$
\Delta=\tau_{2}-\tau_{1}
$$

between two population totals $\tau_{1}$ and $\tau_{2}$ from waves 1 and 2 , where $\tau_{\ell}=\sum_{k \in \mathcal{U}} y_{\ell ; k}$. Here, $\mathcal{U}$ denotes the population of size $N$, assumed to be the same at both waves. It is possible to extend the approach we proposed for other measures of changes, such as relative change or change between means.

Suppose that the population is split into $H$ strata: $U_{1}, \ldots, U_{h}, \ldots, U_{H}$. Suppose that two samples are selected from each strata according to the rotating design described in Figure 1. Let $z_{\ell ; i}^{(h)}$ be the wave-strata sample indicator variable (Berger and Priam, 2016), defined by

$$
z_{\ell ; k}^{(h)}=\left\{\begin{array}{ll}
1 & \text { when } k \in U_{h} \text { and } k \in s_{\ell}, \\
0 & \text { otherwise }
\end{array} \quad(h=1, \ldots, H \quad \text { and } \ell=1,2) .\right.
$$


The values of $z_{\ell ; i}^{(h)}$ are assumed to be known for all $k \in s_{\ell}$. We also consider the wave indicator defined by

$$
z_{\ell ; k}=\sum_{h=1}^{H} z_{\ell ; k}^{(h)}=\left\{\begin{array}{cl}
1 & \text { when } k \in s_{\ell} \\
0 & \text { otherwise }
\end{array}\right.
$$

When we have a single stratum, $H=1$ and $z_{\ell ; i}^{(1)}=z_{\ell ; k}$.

In Section 3, we introduce the uniform non-response mechanism for rotating sampling designs and we show how random hot-deck imputed values can be used to compensate for item non-response. In Section 4, we propose to use a reverse approach (Fay, 1991) to estimate the variance of the imputed estimator of change. The proposed variance estimator depends on a covariance matrix which shall be estimated using a multivariate (general) linear regression approach described in Section 6. In Section 7 , we extend the variance estimator proposed to missing at random response mechanism (MAR) involving multiple imputation-classes. In Section 8.2, we consider a missing not at random (MNAR) response mechanism. In Section 8, a simulation study illustrates our findings.

\section{Non-response}

The main objective of this article is to address the problem of variance estimation under non-response rather than the non-response issue. Little has been done on variance estimation of change. However, there are many design-based variance estimators of cross-sectional estimates (e.g. Wolter, 2007). The use of models to address nonresponse is also popular. A model-assisted approach can be found in Deville and Särndal (1994); Fay (1994); Steel and Fay (1995); Särndal and Lundström (2005). A 
Bayesian treatment of imputation can be found for example in Rubin (1987). See Brick and Montaquila (2009) for a wide discussion on non-response. A discussion on which inference-approach to use for non-response in surveys can be found in Haziza (2009). These approaches deal with cross-sectional estimators, and cannot be directly implemented with estimators of changes.

We propose to use a design-based approach combined with random hot-deck imputation. A recent review on cross-sectional hot-deck imputation can be found in Andridge and Little (2010). The random hot-deck imputation has the advantage of guaranteeing unbiased estimation of population distributions (Rao and Shao, 1992). The approach proposed is also valid under deterministic regression imputation.

Due to non-response, some of the values $y_{\ell ; k}$ can be missing in each sample $s_{\ell}$, $(\ell=1,2)$. We propose to impute these missing values. Let

$$
a_{\ell ; k}=\left\{\begin{array}{ll}
1 & \text { if } y_{\ell ; k} \text { is observed, and } k \in s_{\ell}, \\
0 & \text { if } y_{\ell ; k} \text { is missing due to non-response, and } k \in s_{\ell},
\end{array} \quad(\ell=1,2)\right.
$$

The distribution of the random variables $a_{\ell ; k}$ represents the response mechanism for the wave $\ell$. We assume that the values $a_{\ell ; k}$ are known for all $k \in s_{\ell}$. To simplify, we use the same notation for the random variables and their observed values.

As far as the response mechanism is concerned, we consider the usual crosssectional design-based assumption below (e.g. Fay, 1991; Rao and Shao, 1992; Rao and Sitter, 1995; Shao and Steel, 1999), but adapted for rotating sampling designs.

Assumption 1 (single imputation-class). The response probability for the variable of interest in each wave is uniform on $\mathcal{U}$ and it is strictly positive (i.e. $P\left\{a_{\ell ; k}=1\right\}>0$ ). The $a_{\ell ; k^{\prime}}$ and $a_{\ell ; k^{\prime \prime}}$ are independent for all $k^{\prime} \neq k^{\prime \prime}$ where $k^{\prime}, k^{\prime \prime} \in s_{\ell}$. The responses between waves can be dependent 
that is, $a_{\ell^{\prime} ; k^{\prime}}$ and $a_{\ell^{\prime \prime} ; k^{\prime}}$ may be dependent, where $k^{\prime} \in s_{\ell^{\prime}}$ and $k^{\prime} \in s_{\ell^{\prime \prime}}$. The imputation is conducted independently within waves.

The Assumption 1 implies a missing completely at random (MCAR) response mechanism. The missing at random (MAR) response mechanisms are covered in Section 7. In Section 8.2, we consider a simulation study with a missing not at random (MNAR) response mechanism.

The setting of a single imputation class is the simplest case when handling nonresponse. However, the assumption may be considered unrealistic (e.g. Rao and Shao, 1992, p. 818). We therefore also consider, in Section 7, multiple imputation-classes where values are imputed within imputation classes (e.g. Haziza and Beaumont, 2007). First, we show how the proposed approach can be implemented when we have a single imputation class. In Section 7 we extend the approach proposed under a multiple imputation-classes setting (see Assumption 2).

\subsection{The imputed estimator of change}

Suppose that the change $\Delta$ in (1) is estimated by

$$
\widehat{\Delta}^{I}=\widehat{\tau}_{2}^{I}-\widehat{\tau}_{1}^{I},
$$

where

$$
\widehat{\tau}_{\ell}^{I}=\sum_{k \in \tilde{s}} \frac{y_{\ell ; k}^{I}}{\pi_{\ell ; k}} \quad(\ell=1,2)
$$

is the cross-sectional imputed Narain (1951); Horvitz and Thompson (1952) estimators at wave $\ell$. Here $y_{\ell ; k}^{I}$ is defined by

$$
y_{\ell ; k}^{I}=z_{\ell ; k}\left\{\left(1-a_{\ell ; k}\right) y_{\ell ; k}^{*}+a_{\ell ; k} y_{\ell ; k}\right\},
$$


where $y_{\ell ; k}^{*}$ denotes an imputed value which depends on the imputation technique. For example, in what follows $y_{\ell ; k}^{*}$ is defined by random hot-deck imputation, although the approach proposed can be generalised for other imputation techniques. The deterministic mean imputation is a particular case of hot-deck imputation. Note that the imputation is only used for missing data due to non-response, and not to impute the values $y_{2 ; k}$ of $k \in s_{1} \backslash s_{2}$ and $y_{1 ; k}$ of $k \in s_{2} \backslash s_{1}$ which are the non-available values of the units that rotate in and out.

\subsection{Random hot-deck imputation}

With random hot-deck imputation, the values $y_{\ell ; k}^{*}$ used in equation (6) are,

$$
\begin{aligned}
& y_{\ell ; k}^{*}=\widehat{\mu}_{\ell}^{r}+e_{\ell ; k}, \\
& e_{\ell ; k}=y_{\ell ; j}-\widehat{\mu}_{\ell}^{r},
\end{aligned}
$$

where $j$ is a donor selected with-replacement with probabilities $p_{\ell ; k}=\breve{a}_{\ell ; k} / \widehat{N}_{\ell}^{r}$ from the sample of respondents $s_{\ell}^{r}=\left\{k: z_{\ell ; k}=1\right.$ and $\left.a_{\ell ; k}=1\right\}$. Here $\widehat{\mu}_{\ell}^{r}=\widehat{\tau}_{\ell}^{r} / \widehat{N}_{\ell}^{r}$ is the estimator of the respondents' mean, $\widehat{\tau}_{\ell}^{r}=\sum_{k \in \tilde{s}} \breve{y}_{\ell ; k}$ is the estimator of the respondents' totals, and $\widehat{N}_{\ell}^{r}=\sum_{k \in \tilde{s}} \breve{s}_{\ell ; k}$ is the estimator of the number of respondents for waves $\ell=1,2$; with $\tilde{s}=s_{1} \cup s_{2}$, and where

$$
\begin{aligned}
& \breve{y}_{\ell ; k}=\pi_{\ell ; k}^{-1} z_{\ell ; k} a_{\ell ; k} y_{\ell ; k}, \\
& \breve{a}_{\ell ; k}=\pi_{\ell ; k}^{-1} z_{\ell ; k} a_{\ell ; k} .
\end{aligned}
$$

The $\pi_{\ell ; k}$ denotes the first-order inclusion probability of the unit $k$ at wave $\ell$. If $e_{\ell ; k}=0$ in equation (7), then the $y_{\ell ; k}^{*}$ from equation (6) are the deterministic mean imputed values. 


\section{Population variance of the hot-deck imputed es- timator of change}

We propose to estimate the variance of $\widehat{\Delta}^{I}$ defined by equation (4) using a reverse approach for non-response (Fay, 1991; Shao and Steel, 1999). Let $\mathcal{U}_{\ell}^{r}$ be the population of respondents at wave $\ell$, where $\mathcal{U}_{\ell}^{r} \subset \mathcal{U}$. In other words, at both waves, the population is randomly split into a population of respondents and a population of non-respondents according to an unknown response mechanism. Note that the response mechanisms can be such that the set of respondents of the wave 2 depends on the set of respondents at wave 1 . That is, $a_{1 ; k}$ and $a_{2 ; k}$ can be dependent random variables (see Assumption 1).

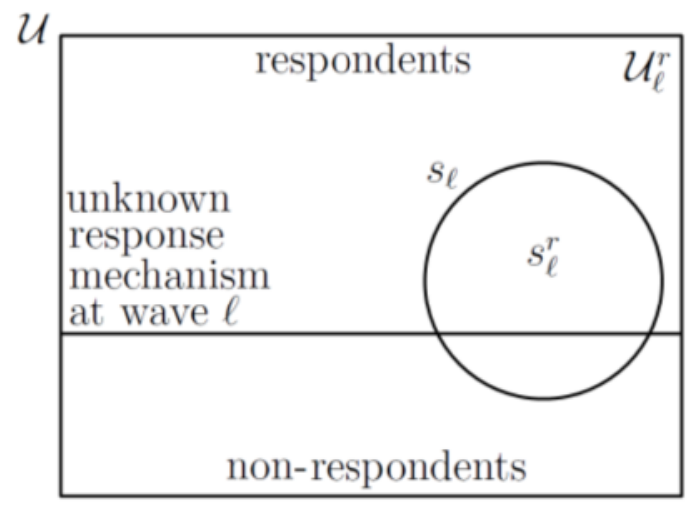

Figure 2: Non-response at wave $\ell=1,2$.

Let $E_{r}\{\cdot\}$ and $V_{r}\{\cdot\}$ denote respectively the expectation and variance operators with respect to the response mechanism. Rotation samples $s_{1}$ and $s_{2}$ are selected from the population $\mathcal{U}$ according to a rotation sampling design (see Section 1). The samples of respondents are given by $s_{\ell}^{r}=\mathcal{U}_{\ell}^{r} \cap s_{\ell},(\ell=1,2)$. Let $E_{d}\{\cdot\}$ and $V_{d}\{\cdot\}$ denote the expectation and the variance operators with respect to the sampling design. Furthermore, we suppose that the random hot-deck imputation described in Section 
3.1 is used. Let $E_{I}\{\cdot\}$ and $V_{I}\{\cdot\}$ denote the expectation and the variance operators with respect to the random imputation.

The overall variance of the imputed estimator of change $\widehat{\Delta}^{I}$ is given by

$$
V\left(\widehat{\Delta}^{I}\right)=A+B+C
$$

which is an overall three stage variance, where

$$
\begin{aligned}
A & =E_{r}\left\{V_{d}\left\{E_{I}\left\{\widehat{\Delta}^{I} \mid S, R\right\} \mid R\right\}\right\}, \\
B & =E_{r}\left\{E_{d}\left\{V_{I}\left\{\widehat{\Delta}^{I} \mid S, R\right\} \mid R\right\}\right\}, \\
C & =V_{r}\left\{E_{d}\left\{E_{I}\left\{\widehat{\Delta}^{I} \mid S, R\right\} \mid R\right\}\right\},
\end{aligned}
$$

with $S=\left\{s_{1}, s_{2}\right\}, R=\left\{s_{1}^{r}, s_{2}^{r}\right\}$. The variance (10) includes the effect of the response mechanism, the sampling design and the imputation. We now focus on each term.

\section{The term A}

Turning to the term A given by equation (11). As $E_{I}\left\{e_{\ell ; k} \mid S, R\right\}=0$, from equation (7), we have that $E_{I}\left\{y_{\ell ; k}^{*} \mid S, R\right\}=\widehat{\mu}_{\ell}^{r}$. Hence, from equations (5) and (6), it can be shown that $E_{I}\left\{\widehat{\tau}_{\ell}^{I} \mid S, R\right\}=\widehat{N}_{\ell} \widehat{\tau}_{\ell}^{r} / \widehat{N}_{\ell}^{r}$. Thus,

$$
E_{I}\left\{\widehat{\Delta}^{I} \mid S, R\right\}=\widehat{N}_{2} \frac{\widehat{\tau}_{2}^{r}}{\widehat{N}_{2}^{r}}-\widehat{N}_{1} \frac{\widehat{\tau}_{1}^{r}}{\widehat{N}_{1}^{r}}
$$

where $\widehat{N}_{\ell}=\sum_{k \in \tilde{s}} \breve{z}_{\ell ; k},(\ell=1,2)$ is an estimator of $N$. Here, $\breve{z}_{\ell ; k}=\pi_{\ell ; k}^{-1} z_{\ell ; k}$. Note that $E_{I}\left\{\widehat{\Delta}^{I} \mid S, R\right\}=f(\widehat{\boldsymbol{\tau}})$, where $f(\cdot)$ is a function of estimated totals $\widehat{\boldsymbol{\tau}}=\left(\widehat{\boldsymbol{\tau}}_{1}^{\top}, \widehat{\boldsymbol{\tau}}_{2}^{\top}\right)^{\top}$, with

$$
\widehat{\tau}_{\ell}=\left(\widehat{N}_{\ell}, \widehat{N}_{\ell}^{r}, \widehat{\tau}_{\ell}^{r}\right)^{\top}
$$


is a vector of Narain-Horvitz-Thompson totals (Narain, 1951; Horvitz and Thompson, 1952). Using a Taylor linearisation (e.g. Särndal, Swensson and Wretman, 1992, §5.5, 5.7 ), we have that

$$
E_{I}\left\{\widehat{\Delta}^{I} \mid S, R\right\}-\Delta \bumpeq \boldsymbol{\nabla}(\boldsymbol{\tau})^{\top}(\widehat{\boldsymbol{\tau}}-\boldsymbol{\tau}),
$$

where

$$
\boldsymbol{\nabla}(\boldsymbol{\tau})=\left(\frac{-\tau_{1}^{r}}{N_{1}^{r}}, \frac{N \tau_{1}^{r}}{\left(N_{1}^{r}\right)^{2}}, \frac{-N}{N_{1}^{r}}, \frac{\tau_{2}^{r}}{N_{2}^{r}}, \frac{-N \tau_{2}^{r}}{\left(N_{2}^{r}\right)^{2}}, \frac{N}{N_{2}^{r}}\right)^{\top},
$$

is the gradient of $f(\boldsymbol{\tau})$ at $\boldsymbol{\tau}=\left(\boldsymbol{\tau}_{1}^{\top}, \boldsymbol{\tau}_{2}^{\top}\right)^{\top}$, with

$$
\boldsymbol{\tau}_{\ell}=\left(N, N_{\ell}^{r}, \tau_{\ell}^{r}\right)^{\top}
$$

Here $\tau_{\ell}^{r}$ is the population total of the variable of interest over the respondents at wave $\ell$; and $N_{\ell}^{r}$ is the total number of respondents in the population at wave $\ell,(\ell=1,2)$.

The expression (16) implies the following approximation

$$
A=E_{r}\left\{V_{d}\left\{E_{I}\left\{\widehat{\Delta}^{I} \mid S, R\right\} \mid R\right\}\right\} \bumpeq \boldsymbol{\nabla}(\boldsymbol{\tau})^{\top} E_{r}\left\{\boldsymbol{V}_{\boldsymbol{d}}(\widehat{\boldsymbol{\tau}} \mid R)\right\} \boldsymbol{\nabla}(\boldsymbol{\tau}),
$$

where $\boldsymbol{V}_{\boldsymbol{d}}(\widehat{\boldsymbol{\tau}} \mid R)$ is the covariance matrix of the vector $\widehat{\boldsymbol{\tau}}$ with respect to the design. Thus, an approximately design-unbiased estimator for (19) is given by

$$
\widehat{A}=\widehat{V}_{d}\left\{E_{I}\left\{\widehat{\Delta}^{I} \mid S, R\right\} \mid R\right\}=\boldsymbol{\nabla}(\widehat{\boldsymbol{\tau}})^{\top} \widehat{\boldsymbol{V}}_{\boldsymbol{d}}(\widehat{\boldsymbol{\tau}} \mid R) \boldsymbol{\nabla}(\widehat{\boldsymbol{\tau}}),
$$

where $\widehat{\boldsymbol{V}}_{\boldsymbol{d}}(\widehat{\boldsymbol{\tau}} \mid R)$ is the approximately design-unbiased estimator of the covariance $\boldsymbol{V}_{\boldsymbol{d}}(\widehat{\boldsymbol{\tau}} \mid R)$. The estimator $\widehat{\boldsymbol{V}}_{\boldsymbol{d}}(\widehat{\boldsymbol{\tau}} \mid R)$ is defined below in equation (28). Note that in equation (20), the $a_{\ell ; k}$ 's are treated as fixed quantities, as $\left.\widehat{V}_{d}\left\{E_{I}\left\{\widehat{\Delta}^{I} \mid S, R\right\} \mid R\right)\right\}$ is a conditional variance given $R$. 


\section{The term B}

We now turn to the term B given by expression (12). Under Assumption 1, we have,

$$
\begin{aligned}
B & =E_{r}\left\{E_{d}\left\{\sum_{\ell=1}^{2} V_{I}\left\{\hat{\tau}_{\ell}^{I} \mid S, R\right\}\right\}\right\} \\
& =E_{r}\left\{E_{d}\left\{\sum_{h=1}^{H} \sum_{\ell=1}^{2} V_{I}\left\{y_{\ell ; k}^{*} \mid S, R\right\} \sum_{k \in \tilde{s}} \frac{z_{\ell ; k}^{(h)}}{\pi_{\ell ; k}^{2}}\left(1-a_{\ell ; k}\right)\right\}\right\}
\end{aligned}
$$

with $V_{I}\left\{y_{\ell ; k}^{*} \mid S, R\right\}=V_{I}\left\{e_{\ell ; k} \mid S, R\right\}=\sum_{k \in \tilde{s}} a_{\ell ; k} p_{\ell ; k} e_{\ell ; k}^{2}$ as $E_{I}\left\{e_{\ell ; k} \mid S, R\right\}=0$. Note that, under deterministic mean imputation, we have $V_{I}\left\{\widehat{\Delta}^{I} \mid S, R\right\}=0$. This is also the case for regression imputation.

The expression (21) implies that an unbiased estimator of expression (12) is given by

$$
\widehat{B}=\widehat{V}_{I}\left\{\widehat{\Delta}^{I} \mid S, R\right\}=\sum_{h=1}^{H} \sum_{\ell=1}^{2} V_{I}\left\{y_{\ell ; k}^{*} \mid S, R\right\} \sum_{k \in \tilde{s}} \frac{z_{\ell ; k}^{(h)}}{\pi_{\ell ; k}^{2}}\left(1-a_{\ell ; k}\right) .
$$

Note that we use the same notation for the random variables $e_{\ell ; k}$ 's and their observed values.

\section{The term C}

We now turn to the term $\mathrm{C}$ given by equation (13). Let

$$
\Upsilon_{\ell}=E_{d}\left\{E_{I}\left\{\hat{t}_{\ell}^{I} \mid S, R\right\} \mid R\right\}=N_{\ell} \frac{\tau_{\ell}^{r}}{N_{\ell}^{r}}
$$

We have from equation (4) that $E_{d}\left\{E_{I}\left\{\widehat{\Delta}^{I} \mid S, R\right\} \mid R\right\}=\Upsilon_{2}-\Upsilon_{1}$. Hence, from equation (13),

$$
C=V_{r}\left(\Upsilon_{1}\right)+V_{r}\left(\Upsilon_{2}\right)-2 \operatorname{Corr}_{r}\left(\Upsilon_{1}, \Upsilon_{2}\right) \sqrt{V_{r}\left(\Upsilon_{1}\right) V_{r}\left(\Upsilon_{2}\right)},
$$


where $\operatorname{Corr}_{r}(\cdot)$ denotes the correlation operator with respect to the response mechanism. Here, $V_{r}\left(\Upsilon_{\ell}\right)=V_{r}\left\{E_{d}\left\{E_{I}\left\{\hat{t}_{\ell}^{I} \mid S, R\right\} \mid R\right\}\right\}$ is the cross-sectional variance for wave $\ell$ under the response mechanism given the random imputation and the sampling design.

We recall from Shao and Steel (1999, pp. 256, 257) that the cross-sectional variances $V_{r}\left(\Upsilon_{\ell}\right)$ are of order $\mathcal{O}\left(N_{\ell}\right)$ implying $C=\mathcal{O}\left(N_{\ell}\right)$, because the correlation $\operatorname{Corr}_{r}\left(\Upsilon_{1}, \Upsilon_{2}\right)$ is between -1 and 1 in equation (23). Given standard assumptions for linearised variances of functions of totals (e.g. Robinson and Särndal, 1983; Särndal et al., 1992, secs. 5.5, 5.7), the linearised version of the term $A$ from equation (19) is of order $\mathcal{O}\left(N_{\ell}^{2} / n\right)$, which is the dominant term of the overall variance $V\left(\widehat{\Delta}^{I}\right)$ from equation (10). Furthermore, $C / A=\mathcal{O}\left(n / N_{\ell}\right)$. Thus, for negligible $n / N_{\ell}$ the contribution of $C$ to equation (10) is negligible (e.g. Haziza, 2009, pp. 238-240). Thus,

$$
V\left(\widehat{\Delta}^{I}\right) \bumpeq A+B
$$

From equation (23), we note that the response mechanisms can be dependent between waves. In other words, even if $a_{1 ; k}$ and $a_{2 ; k}$ are dependent, the equation (24) still holds.

\section{The proposed variance estimator}

We proposed to estimate the variance of the imputed estimator (4) by

$$
\widehat{V}\left\{\widehat{\Delta}^{I}\right\}=\widehat{A}+\widehat{B}=\widehat{V}_{d}\left\{E_{I}\left\{\widehat{\Delta}^{I} \mid S, R\right\} \mid R\right\}+\widehat{V}_{I}\left\{\widehat{\Delta}^{I} \mid S, R\right\},
$$

where $\widehat{V}_{d}\left\{E_{I}\left\{\widehat{\Delta}^{I} \mid S, R\right\} \mid R\right\}$ and $\widehat{V}_{I}\left\{\widehat{\Delta}^{I} \mid S, R\right\}$ are defined by expressions (20) and (22). In section 6 , we propose a multivariate (or general) linear regression model to estimate the covariance matrix $\boldsymbol{V}_{\boldsymbol{d}}(\widehat{\boldsymbol{\tau}} \mid R)$ involved in the estimator (20) (Berger and Priam, 
2010, 2016). The proposed estimator (25) is an approximately unbiased estimator of the variance $V\left(\widehat{\Delta}^{I}\right)$ given by equation (10), as the overall expectation of the estimator (25) is given by

$$
\begin{aligned}
E_{r}\left\{E_{d}\left\{E_{I}\left\{\widehat{V}\left(\widehat{\Delta}^{I}\right) \mid S, R\right\} \mid R\right\}\right\}= & E_{r}\left\{E_{d}\left\{E_{I}\left\{\widehat{V}_{d}\left(E_{I}\left\{\widehat{\Delta}^{I} \mid S, R\right\} \mid R\right) \mid S, R\right\} \mid R\right\}\right\} \\
& +E_{r}\left\{E_{d}\left\{E_{I}\left\{V_{I}\left\{\widehat{\Delta}^{I} \mid S, R\right\}\right\} \mid R\right\}\right\} \\
\bumpeq & E_{r}\left\{V_{d}\left\{E_{I}\left\{\widehat{\Delta}^{I} \mid S, R\right\} \mid R\right\}\right\} \\
& +E_{r}\left\{E_{d}\left\{V_{I}\left\{\widehat{\Delta}^{I} \mid S, R\right\} \mid R\right\}\right\} \\
\bumpeq & V\left(\widehat{\Delta}^{I}\right),
\end{aligned}
$$

by using the result (24) and the fact that the estimator (20) does not depends on the $e_{\ell ; k}$ 's for $\ell=1,2$.

The advantages of the proposed variance estimator (25) are that it is approximately unbiased under the unknown response mechanisms and that it does not involve the estimation of the response probabilities. Moreover, note that the estimator (25)

can be generalised for other types of imputation, as long as $E_{I}\left\{\widehat{\Delta}^{I} \mid S, R\right\}$ is a function of Narain-Horvitz-Thompson estimators of totals. In this situation, the gradient (17) would have a different expression depending on the imputation considered.

\section{Estimation of the covariance using the multivari- ate regression approach}

We derive an estimator for the covariance matrix $\boldsymbol{V}_{\boldsymbol{d}}(\widehat{\boldsymbol{\tau}} \mid R)$ in equation (19) under the rotation sampling design. Note that this covariance is not straightforward to estimate because it involves a covariance between all the components of $\widehat{\tau}$ defined 
from different overlapping samples, $s_{1}$ and $s_{2}$. Several estimators can be used (e.g. Kish, 1965; Tam, 1984; Holmes and Skinner, 2000; Nordberg, 2000; Berger, 2004; Qualité and Tillé, 2008; Wood, 2008; Goga, Deville and Ruiz-Gazen, 2009; Münnich and Zins, 2011; Knottnerus and van Delden, 2012). We propose to use a multivariate (or general) linear regression model to estimate this covariance matrix (Berger and Priam, 2010, 2016).

Consider the following $\tilde{n} \times 6$ matrix $\breve{\boldsymbol{Y}}_{(\tilde{n} \times 6)}=\left(\breve{\boldsymbol{y}}_{1}, \ldots, \breve{\boldsymbol{y}}_{k}, \ldots, \breve{\boldsymbol{y}}_{\tilde{n}}\right)^{\top}$, where $\tilde{n}=$ $\#\left(s_{1} \cup s_{2}\right), \breve{\boldsymbol{y}}_{k}=\left(\breve{\boldsymbol{y}}_{1 k}, \breve{\boldsymbol{y}}_{2 k}\right)$ and

$$
\breve{\boldsymbol{y}}_{\ell k}=\left(\breve{z}_{\ell ; k}, \breve{,}_{\ell ; k}, \breve{y}_{\ell ; k}\right)^{\top}
$$

with $\breve{z}_{\ell ; k}=\pi_{\ell ; k}^{-1} z_{\ell ; k}$. Here, $z_{\ell ; k}, \breve{a}_{\ell ; k}$ and $\breve{y}_{\ell ; k}$ are defined by the expressions (3), (9) and (8), with $\ell=1,2$. Consider the following multivariate (general) regression model

$$
\breve{Y}=Z_{s} \boldsymbol{\alpha}+\varepsilon
$$

where $\boldsymbol{\alpha}$ is a $3 \times 6$ matrix of regression parameters, the residuals $\varepsilon$ have a $6 \times 6$ covariance matrix $\boldsymbol{\Sigma}$, and $\boldsymbol{Z}_{s}$ is a $\tilde{n} \times 3$ design matrix which specifies the fixed-size constraints of the rotation design. The matrix $\boldsymbol{Z}_{s}$ is defined by $\boldsymbol{Z}_{s}=\left(\boldsymbol{z}_{1}, \ldots, \boldsymbol{z}_{k}, \ldots, \boldsymbol{z}_{\tilde{n}}\right)^{\top}$ with

$$
\boldsymbol{z}_{k}=\left(z_{1 ; k}^{(1)}, \ldots, z_{1 ; k}^{(H)}, z_{2 ; k}^{(1)}, \ldots, z_{2 ; k}^{(H)}, z_{1 ; k}^{(1)} \times z_{2 ; k}^{(1)}, \ldots, z_{1 ; k}^{(H)} \times z_{2 ; k}^{(H)}\right)^{\top}
$$

With a single stratum, $\boldsymbol{z}_{k}=\left(z_{1 ; k}, z_{2 ; k}, z_{1 ; k} \times z_{2 ; k}\right)^{\top}$. The model (27) belongs to the class of general linear model. In fact, the model (27) is also a multivariate analysis of variance (MANOVA) model, as the covariates are all dummy variables. Note that we have $\sum_{k \in \tilde{s}} z_{\ell ; k}^{(h)}=n_{h}, \sum_{k \in \tilde{s}} z_{1 ; k}^{(h)} z_{2 ; k}^{(h)}=n_{h ; 12}$, where $n_{h}$ is the sample size of the $h$-th stratum and $n_{h ; 12}$ is the number of wave 1 and 2 units which belong to the $h$-th 
stratum. These sums are the sample size restriction imposed on the samples. Thus, by using the design variables as covariates in the model (27), we implicitly condition on them. This takes into account the fixed size constraints in the estimation of the covariance (see Berger and Priam, 2010, 2016). Note that the model (27) includes the within strata interactions between the variable $z_{1 ; k}^{(h)}$ and $z_{2 ; k}^{(h)}$. These interactions capture the rotation of the sampling design which is represented by the constraint $\sum_{k \in \tilde{s}} z_{1 ; k}^{(h)} z_{2 ; k}^{(h)}=n_{h ; 12}$.

To estimate $\boldsymbol{V}_{\boldsymbol{d}}(\widehat{\boldsymbol{\tau}} \mid R)$, Berger and Priam $(2010,2016)$ proposed the estimator

$$
\widehat{V}_{d}(\widehat{\tau} \mid R)=\widehat{D}^{\top} \widehat{\Sigma} \widehat{D}
$$

where the matrix $\widehat{\mathbf{\Sigma}}$ is the Ordinary Least Squares residual covariance matrix estimate from the model (27) and $\widehat{\boldsymbol{D}}$ is a diagonal matrix with the diagonal elements: $\left\{\widehat{V}\left(\widehat{\tau}_{q} \mid R\right) / \widehat{\Sigma}_{q q}\right\}^{1 / 2}$, where $\widehat{V}\left(\widehat{\tau}_{q} \mid R\right)$ is a design-based variance estimator of the $q$-th component of $\widehat{\boldsymbol{\tau}}$ and $\widehat{\Sigma}_{q q}$ is the $q$-th diagonal component of $\widehat{\Sigma}$. Any approximately unbiased variance estimator can be used to calculate $\widehat{V}\left(\widehat{\tau}_{q} \mid R\right)$. Note that the variance matrix (28) is positive definite, as $\widehat{\Sigma}$ is always positive definite. Hence, the proposed variance estimator (25) is always positive.

Using Berger and Priam; Berger and Priam's (2010; 2016) results, we have that the estimator (28) is an approximately design unbiased estimator for $\boldsymbol{V}_{\boldsymbol{d}}(\widehat{\boldsymbol{\tau}} \mid R)$ when the finite population corrections are negligible, even when model (27) does not fit the data (Berger and Priam, 2010, 2016).

In a series of simulations based on the Swedish Labour Force Survey, Andersson, Andersson and Lundquist (2011a); Andersson, Andersson and Lundquist (2011b) showed that under full response, the estimator that was proposed by Berger (2004) gives more accurate estimates than standard variance estimators (e.g. Tam, 1984; 
Qualité and Tillé, 2008) when we are interested in change between strata domains. Berger and Priam $(2010,2016)$ showed that the estimator that was proposed by Berger (2004) reduces to the variance (28) when the sampling fractions are negligible.

\title{
7 Multiple imputation-classes
}

We now consider the situation when the response mechanism is not uniform. Hence, instead of the Assumption 1, we have the following assumption:

\begin{abstract}
Assumption 2 (multiple imputation-classes). The population $\mathcal{U}$ can be divided into $C_{1}$ imputation classes for wave 1 and $C_{2}$ imputation classes for wave 2. The response probability for the variable of interest is uniform within wave-class combinations and it is strictly positive. The units' responses within and across classes are independent; and responses between waves can be dependent.
\end{abstract}

The Assumption 2 holds under missing at random (MAR) response mechanism (given the set of classes).

Let $\mathcal{U}_{\ell ; 1}, \ldots, \mathcal{U}_{\ell ; c}, \ldots, \mathcal{U}_{\ell ; C_{\ell}}$ be the $C_{\ell}$ class of wave $\ell$. Let $b_{\ell ; k}^{(c)}$ be the following imputation classes indicator for wave $\ell$.

$$
b_{\ell ; k}^{(c)}= \begin{cases}1 & \text { if } k \in \mathcal{U}_{\ell ; c} \\ 0 & \text { otherwise }\end{cases}
$$

where $c=1, \ldots, C_{\ell}$. The random hot-deck imputed values $y_{\ell ; k}^{I}$ from equation (6) is now given by $y_{\ell ; k}^{*}=\sum_{c=1}^{C} b_{\ell ; k}^{(c)}\left(\widehat{\mu}_{\ell}^{r(c)}+e_{\ell ; k}^{(c)}\right)$ where $e_{\ell ; k}^{(c)}=y_{\ell ; j}-\widehat{\mu}_{\ell}^{r(c)}$ instead of expression (7), where $j$ is a donor selected with-replacement with probabilities $p_{\ell ; k}=$ $b_{\ell ; k}^{(c)} \breve{a}_{\ell ; k} / \widehat{N}_{\ell}^{r(c)}$ from the sample $s_{\ell}^{r(c)}$ of respondents of class $c$. Here, $s_{\ell}^{r(c)}=\left\{k: z_{\ell ; k}=\right.$ 
$\left.1, a_{\ell ; k}=1, b_{\ell ; k}^{(c)}=1\right\}$ and

$$
\begin{aligned}
\widehat{\mu}_{\ell}^{r(c)} & =\frac{\widehat{\tau}_{\ell}^{r(c)}}{\widehat{N}_{\ell}^{r(c)}}, \\
\widehat{\tau}_{\ell}^{r(c)} & =\sum_{k \in \tilde{s}} b_{\ell ; k}^{(c)} \breve{y}_{\ell ; k}, \\
\widehat{N}_{\ell}^{r(c)} & =\sum_{k \in \tilde{s}} b_{\ell ; k}^{(c)} \breve{a}_{\ell ; k},
\end{aligned}
$$

are respectively the estimates of the respondents' means, totals and number of respondents for each wave-class combination $\left(c=1,2, \ldots, C_{\ell} ; \ell=1,2\right)$. Note that under multiple-classes deterministic mean imputation, we have $e_{\ell ; k}^{(c)}=0$.

With multiple imputation-classes the population variance $V\left(\widehat{\Delta}^{I}\right)$ from equation (10) is different. In the term A from expression (11), the equations (14), (15), (17) and (18) are replaced by,

$$
\begin{aligned}
& E_{I}\left\{\widehat{\Delta}^{I} \mid S, R\right\}=\widehat{N}_{2} \frac{\sum_{c=1}^{C_{2}} \widehat{\tau}_{2}^{r(c)}}{\sum_{c=1}^{C_{2}} \widehat{N}_{2}^{r(c)}}-\widehat{N}_{1} \frac{\sum_{c=1}^{C_{1}} \widehat{\tau}_{1}^{r(c)}}{\sum_{c=1}^{C_{1}} \widehat{N}_{1}^{r(c)}}, \\
& \widehat{\boldsymbol{\tau}}_{\ell}=\left(\widehat{N}_{\ell}, \widehat{N}_{\ell}^{r(1)}, \ldots, \widehat{N}_{\ell}^{r\left(C_{1}\right)}, \widehat{\tau}_{\ell}^{r(1)}, \ldots, \widehat{\tau}_{\ell}^{r\left(C_{2}\right)}\right)^{\top}, \\
& \boldsymbol{\nabla}(\boldsymbol{\tau})=(\frac{-\tau_{1}^{r}}{N_{1}^{r}}, \underbrace{\frac{N \tau_{1}^{r}}{\left(N_{1}^{r}\right)^{2}}, \ldots}_{C_{1} \text { times }}, \underbrace{\frac{-N}{N_{1}^{r}}, \ldots}_{C_{1} \text { times }}, \frac{\tau_{2}^{r}}{N_{2}^{r}}, \underbrace{\frac{-N \tau_{2}^{r}}{\left(N_{2}^{r}\right)^{2}}, \ldots}_{C_{2} \text { times }}, \underbrace{\frac{N}{N_{2}^{r}}, \ldots}_{C_{2} \text { times }})^{\top} \\
& \boldsymbol{\tau}_{\ell}=\left(N, N_{\ell}^{r(1)}, \ldots, N_{\ell}^{r\left(C_{\ell}\right)}, \tau_{\ell}^{r(1)}, \ldots, \tau_{\ell}^{r\left(C_{\ell}\right)}\right)^{\top},
\end{aligned}
$$

with $\tau_{\ell}^{r}=\sum_{c=1}^{C_{\ell}} \tau_{\ell}^{r(c)}$ and $N_{\ell}^{r}=\sum_{c=1}^{C_{\ell}} N_{\ell}^{r(c)}$, where $\tau_{\ell}^{r(c)}$ and $N_{\ell}^{r(c)}$ are, respectively, the respondents' population totals of the variable $y_{k}$ and the number of respondents at each wave-class combination, $\left(c=1,2, \ldots, C_{\ell} ; \ell=1,2\right)$. 
Under Assumption 2, we have that

$$
\widehat{V}_{I}\left\{\widehat{\Delta}^{I} \mid S, R\right\}=\sum_{\ell=1}^{2} \sum_{c=1}^{C_{\ell}} \sum_{k \in \tilde{s}} a_{\ell ; k} p_{\ell ; k}\left\{e_{\ell ; k}^{(c)}\right\}^{2} \sum_{k^{\prime} \in \tilde{s}} \frac{b_{\ell ; k^{\prime}}^{(c)} z_{\ell ; k^{\prime}}}{\pi_{\ell ; k^{\prime}}^{2}}\left(1-a_{\ell ; k^{\prime}}\right) .
$$

Thus, the estimator proposed is given by expression (25) where $\widehat{V}_{d}\left\{E_{I}\left\{\widehat{\Delta}^{I} \mid S, R\right\} \mid R\right\}$ and $V_{I}\left\{\widehat{\Delta}^{I} \mid S, R\right\}$ are now given by equations (20) and (33). The quantities $E_{I}\left\{\widehat{\Delta}^{I} \mid S, R\right\}$, $\widehat{\boldsymbol{\tau}}_{\ell}, \boldsymbol{\nabla}(\boldsymbol{\tau})$, and $\boldsymbol{\tau}_{\ell}$, are now given by equations (29), (30), (31) and (32).

As in Section 6, the covariance matrix $\boldsymbol{V}_{\boldsymbol{d}}(\widehat{\boldsymbol{\tau}} \mid R)$ in equation (19) can be estimated using a multivariate (or general) linear regression model. With multiple imputationclasses, the model (27) now uses a $\tilde{n} \times\left(2+2 C_{1}+2 C_{2}\right)$ matrix $\breve{\boldsymbol{Y}}=\left(\breve{\boldsymbol{y}}_{1}, \ldots, \breve{\boldsymbol{y}}_{k}, \ldots, \breve{\boldsymbol{y}}_{\tilde{n}}\right)^{\top}$ where $\breve{\boldsymbol{y}}_{k}=\left(\breve{\boldsymbol{y}}_{1 k}, \breve{\boldsymbol{y}}_{2 k}\right)$, with

$$
\breve{\boldsymbol{y}}_{\ell k}=\left(\breve{z}_{\ell ; k}, b_{\ell ; k}^{(1)} \breve{a}_{\ell ; k}, \ldots, b_{\ell ; k}^{\left(C_{\ell}\right)} \breve{a}_{\ell ; k}, b_{\ell ; k}^{(1)} \breve{y}_{\ell ; k}, \ldots, b_{\ell ; k}^{\left(C_{\ell}\right)} \breve{y}_{\ell ; k}\right)^{\top}
$$

replaces the expression (26). Now $\boldsymbol{\alpha}$ is a $3 \times\left(2+2 C_{1}+2 C_{2}\right)$ matrix and $\boldsymbol{\Sigma}$ is a $\left(2+2 C_{1}+2 C_{2}\right) \times\left(2+2 C_{1}+2 C_{2}\right)$ matrix.

The variance estimator (28) is based on the implicit assumption that the class indicator $b_{\ell ; k}^{(c)}$ are constants defined at population level. In other words, the $b_{\ell ; k}^{(c)}$ do not depend on the samples selected. For example, this is the case when we use the strata as imputation classes; that is, the $b_{\ell ; k}^{(c)}$ are the strata indicators $z_{\ell ; i}^{(h)}$ defined by (2). In the Section 8.2 , we will consider the situation when the $b_{\ell ; k}^{(c)}$ are defined form the sampled data; that is, the $b_{\ell ; k}^{(c)}$ are random variable because they depend on the samples selected. In this case, the variance estimator (28) does not take the randomness of $b_{\ell ; k}^{(c)}$ into account. In the simulation study of Section 8.2, we did not observe major impacts of this randomness. 


\section{Simulation study}

\subsection{Labor Force Population}

We use the Labor Force Population dataset from Valliant, Dorfman and Royall (2000, Appendix B.5) available at the John Wiley worldwide website. The dataset is duplicated 50 times to obtain a large population suitable for different levels of rotation and small sampling fractions in the sampling design. We consider two variables: the weekly wages and the hours worked per week $(H W)$. The units with the value 99 for the weekly wage and 999 for the hours worked per week were removed from the population frame. These units were not treated as missing. We obtain a population frame of size $N=23550$. The target variables $y_{1 ; k}$ and $y_{2 ; k}$ are given by,

$$
\begin{aligned}
& y_{1 ; k}=\text { Weekly wages }, \\
& y_{2 ; k}=y_{1 ; k}+\sqrt{y_{1 ; k}}+\psi_{k},
\end{aligned}
$$

where $\psi_{k}$ denotes randomly generated values according to a Normal distribution $N\left(0,5^{2}\right)$. The true absolute change between the two wave totals is given by $\Delta=$

377960.66. We estimate $\Delta$ by the hot-deck imputed point estimator $\widehat{\Delta}^{I}$ defined by equation (4). The first wave sample $s_{1}$ is selected using the Rao (1965) and Sampford (1967) unequal probability sampling design. We consider two scenarios for the inclusion probabilities: the $\pi_{1 ; k}$ are constant $\left(\pi_{1 ; k}=n / N\right)$, and the $\pi_{1 ; k}$ are proportional to the variable hours worked per week which has values all larger than 5 . We consider that we have a single stratum.

For the second wave sample $s_{2}$ we select a simple random sample of $n_{12}$ units taken from $s_{1}$, where $g=n_{12} / n=\{0.40,0.60,0.80,0.95\}$, and a sample of $n-n_{12}$ units from $\mathcal{U} \backslash s_{1}$ selected with probabilities proportional to $\pi_{2 ; k}=\pi_{1 ; k} /\left(1-\pi_{1 ; k}\right)$. We have that $\pi_{2 ; k} \bumpeq \pi_{1 ; k}$ (Berger and Priam, 2016). 
Table 1: RB, RRMSE and Coverage of $95 \%$ confidence interval of the variance estimators. Hot-deck imputed point estimator $\widehat{\Delta}^{I} . \pi_{1 ; k}=n / N$.

\begin{tabular}{|c|c|c|c|c|c|c|c|c|c|}
\hline \multirow[t]{3}{*}{$q_{1 ; k}$} & \multirow[t]{3}{*}{$q_{2 ; k}$} & \multirow{3}{*}{$\begin{array}{c}g \\
(\%)\end{array}$} & \multirow[t]{2}{*}{$f$} & \multicolumn{2}{|c|}{$\mathrm{RB}$} & \multicolumn{2}{|c|}{ RRMSE } & \multicolumn{2}{|c|}{ Coverage } \\
\hline & & & & Prop. & Naïve & Prop. & Naïve & Prop. & Naïve \\
\hline & & & $(\%)$ & & $(\%)$ & & $(\%)$ & $(\%)$ & $(\%)$ \\
\hline \multirow[t]{16}{*}{0.70} & 0.86 & 40 & 0.5 & -2.8 & -33.8 & 15.5 & 35.3 & 95.0 & 88.7 \\
\hline & & & 1.0 & -0.7 & -32.3 & 11.2 & 33.2 & 94.8 & 89.3 \\
\hline & & & 1.5 & -0.4 & -32.1 & 9.1 & 32.7 & 94.7 & 89.5 \\
\hline & & & 2.0 & -2.7 & -33.7 & 8.2 & 34.1 & 94.6 & 88.8 \\
\hline & & 60 & 0.5 & -1.8 & -31.3 & 17.6 & 33.7 & 94.7 & 89.1 \\
\hline & & & 1.0 & -1.2 & -30.9 & 12.5 & 32.2 & 94.8 & 89.7 \\
\hline & & & 1.5 & -1.1 & -30.8 & 10.2 & 31.7 & 94.7 & 89.2 \\
\hline & & & 2.0 & 0.0 & -30.1 & 8.7 & 30.8 & 94.8 & 89.9 \\
\hline & & 80 & 0.5 & -1.8 & -28.8 & 20.1 & 32.7 & 94.7 & 89.8 \\
\hline & & & 1.0 & -0.4 & -27.5 & 14.4 & 29.8 & 95.0 & 90.4 \\
\hline & & & 1.5 & -0.4 & -27.5 & 11.6 & 29.0 & 95.0 & 90.5 \\
\hline & & & 2.0 & -2.2 & -29.0 & 10.0 & 30.0 & 94.6 & 90.0 \\
\hline & & $\overline{95}$ & 0.5 & -1.8 & -25.3 & 22.8 & 31.9 & 94.8 & 90.8 \\
\hline & & & 1.0 & -1.9 & -25.5 & 16.0 & 29.0 & 94.5 & 90.8 \\
\hline & & & 1.5 & -0.9 & -24.8 & 13.1 & 27.2 & 94.8 & 90.7 \\
\hline & & & 2.0 & -1.6 & -25.3 & 11.2 & 27.0 & 94.7 & 90.9 \\
\hline \multirow[t]{16}{*}{0.90} & 0.92 & 40 & 0.5 & -0.7 & -15.9 & 14.5 & 20.2 & 94.8 & 92.9 \\
\hline & & & 1.0 & 0.2 & -15.2 & 10.2 & 17.6 & 95.3 & 93.2 \\
\hline & & & 1.5 & -2.1 & -17.2 & 8.5 & 18.5 & 94.7 & 92.6 \\
\hline & & & 2.0 & -0.7 & -15.9 & 7.2 & 17.1 & 95.1 & 92.8 \\
\hline & & 60 & 0.5 & 0.4 & -14.4 & 17.2 & 21.0 & 94.9 & 93.2 \\
\hline & & & 1.0 & 0.2 & -14.6 & 12.1 & 18.2 & 94.9 & 92.9 \\
\hline & & & 1.5 & 0.6 & -14.2 & 9.9 & 16.7 & 95.1 & 93.1 \\
\hline & & & 2.0 & 0.0 & -14.8 & 8.5 & 16.7 & 94.8 & 92.7 \\
\hline & & 80 & 0.5 & -2.2 & -15.3 & 21.4 & 25.5 & 94.7 & 93.0 \\
\hline & & & 1.0 & -2.0 & -15.0 & 15.0 & 20.8 & 95.0 & 93.1 \\
\hline & & & 1.5 & -0.2 & -13.7 & 12.3 & 18.2 & 94.8 & 92.9 \\
\hline & & & 2.0 & -1.0 & -14.4 & 10.7 & 17.7 & 94.6 & 92.9 \\
\hline & & 95 & 0.5 & -2.9 & -13.4 & 27.7 & 33.0 & 94.5 & 92.9 \\
\hline & & & 1.0 & -2.4 & -13.2 & 19.4 & 24.8 & 94.5 & 93.2 \\
\hline & & & 1.5 & -1.1 & -12.0 & 15.9 & 21.3 & 95.1 & 93.6 \\
\hline & & & 2.0 & -0.9 & -12.0 & 13.8 & 19.3 & 94.9 & 93.5 \\
\hline
\end{tabular}

Let $a_{1 ; k}=1$ if $u_{1 ; k} \leq q_{1}$ and $a_{1 ; k}=0$ otherwise, where $q_{1}$ is a fixed quantity which specify the response rate at wave 1 , and $u_{1 ; k}$ are independent uniform random variables $U(0,1)$. Let $a_{2 ; k}=1$ if $u_{2 ; k} \leq(0.95) a_{1 ; k}+(0.65)\left(1-a_{1 ; k}\right)$ and $a_{2 ; k}=0$ 
Table 2: RB, RRMSE and Coverage of $95 \%$ confidence interval of the variance estimators. Hot-deck imputed point estimator $\widehat{\Delta}^{I} . \pi_{1 ; k} \propto H W$.

\begin{tabular}{|c|c|c|c|c|c|c|c|c|c|}
\hline \multirow[t]{3}{*}{$q_{1 ; k}$} & \multirow[t]{3}{*}{$q_{2 ; k}$} & \multirow{3}{*}{$\begin{array}{c}g \\
(\%)\end{array}$} & \multirow[t]{2}{*}{$f$} & \multicolumn{2}{|c|}{$\mathrm{RB}$} & \multicolumn{2}{|c|}{ RRMSE } & \multicolumn{2}{|c|}{ Coverage } \\
\hline & & & & Prop. & Naïve & Prop. & Naïve & Prop. & Naïve \\
\hline & & & $(\%)$ & & $(\%)$ & & & $(\%)$ & $(\%)$ \\
\hline \multirow[t]{16}{*}{0.70} & 0.86 & 40 & 0.5 & -1.6 & -29.1 & 32.3 & 52.7 & 94.3 & 88.7 \\
\hline & & & 1.0 & -2.5 & -29.9 & 23.3 & 42.8 & 93.5 & 87.3 \\
\hline & & & 1.5 & -3.4 & -30.5 & 19.0 & 39.7 & 93.0 & 86.8 \\
\hline & & & 2.0 & -1.5 & -29.4 & 16.4 & 36.7 & 92.4 & 86.5 \\
\hline & & 60 & 0.5 & -2.0 & -27.7 & 36.2 & 57.1 & 94.3 & 89.3 \\
\hline & & & 1.0 & -1.2 & -27.5 & 26.1 & 44.0 & 94.2 & 88.9 \\
\hline & & & 1.5 & -0.8 & -27.1 & 21.4 & 39.1 & 94.1 & 88.9 \\
\hline & & & 2.0 & -0.7 & -27.5 & 18.2 & 36.4 & 93.5 & 87.8 \\
\hline & & 80 & 0.5 & -0.1 & -25.6 & 40.7 & 59.2 & 94.8 & 90.4 \\
\hline & & & 1.0 & 0.0 & -25.2 & 29.3 & 45.5 & 94.9 & 89.7 \\
\hline & & & 1.5 & -0.4 & -25.1 & 23.6 & 40.4 & 94.8 & 90.2 \\
\hline & & & 2.0 & -0.6 & -25.8 & 20.4 & 37.1 & 94.5 & 89.7 \\
\hline & & $\overline{95}$ & 0.5 & -1.5 & -24.3 & 43.9 & 63.6 & 94.5 & 90.8 \\
\hline & & & 1.0 & 0.4 & -22.9 & 31.7 & 48.5 & 95.2 & 91.3 \\
\hline & & & 1.5 & 0.5 & -23.4 & 26.0 & 41.4 & 94.9 & 91.2 \\
\hline & & & 2.0 & -0.8 & -24.3 & 22.3 & 38.1 & 94.9 & 90.6 \\
\hline \multirow[t]{16}{*}{0.90} & 0.92 & 40 & 0.5 & -0.5 & -15.5 & 34.3 & 51.2 & 94.1 & 91.7 \\
\hline & & & 1.0 & -1.5 & -15.6 & 23.5 & 37.7 & 93.1 & 90.4 \\
\hline & & & 1.5 & -0.5 & -14.8 & 19.9 & 33.1 & 92.9 & 90.2 \\
\hline & & & 2.0 & -1.7 & -16.0 & 16.9 & 29.7 & 91.8 & 88.9 \\
\hline & & 60 & 0.5 & -0.1 & -14.2 & 41.2 & 61.1 & 94.3 & 92.3 \\
\hline & & & 1.0 & -2.4 & -15.3 & 29.2 & 48.1 & 93.8 & 91.6 \\
\hline & & & 1.5 & 0.2 & -13.4 & 23.6 & 38.1 & 93.9 & 91.3 \\
\hline & & & 2.0 & -1.0 & -14.4 & 20.5 & 34.3 & 93.0 & 90.6 \\
\hline & & 80 & 0.5 & -0.3 & -12.8 & 51.9 & 78.6 & 94.5 & 93.1 \\
\hline & & & 1.0 & -0.3 & -11.7 & 36.3 & 59.9 & 94.7 & 93.1 \\
\hline & & & 1.5 & -1.3 & -12.8 & 29.3 & 49.3 & 94.2 & 92.0 \\
\hline & & & 2.0 & -0.4 & -12.4 & 25.6 & 42.1 & 94.2 & 92.1 \\
\hline & & 95 & 0.5 & -0.8 & -11.5 & 64.3 & 99.0 & 94.7 & 94.4 \\
\hline & & & 1.0 & -1.9 & -11.5 & 44.0 & 71.4 & 94.3 & 93.5 \\
\hline & & & 1.5 & -1.5 & -11.9 & 35.5 & 58.8 & 94.6 & 93.2 \\
\hline & & & 2.0 & -0.8 & -11.3 & 30.7 & 49.7 & 94.6 & 93.4 \\
\hline
\end{tabular}

otherwise, where $u_{2 ; k}$ are independent uniform random variables $U(0,1)$. Note that $a_{1 ; k}$ and $a_{2 ; k}$ are dependent because a respondent at wave 1 is more likely to be also a respondent on wave 2 . The items non-response are imputed using random hot-deck 
as described in subsections 3.1 and 3.2. We consider that we have a single imputation class. A new set of respondents $\left(a_{1 ; k}, a_{2 ; k}\right)$ is generated randomly before each selection of $s_{1}$ and $s_{2}$.

For each simulation, 10000 samples are selected to compute: the empirical relative bias $\mathrm{RB}=\operatorname{Bias}\left(\widehat{\operatorname{var}}\left(\widehat{\Delta}^{I}\right)\right) / \operatorname{var}\left(\widehat{\Delta}^{I}\right)$ where $\operatorname{Bias}\left(\widehat{\operatorname{var}}\left(\widehat{\Delta}^{I}\right)\right)=\mathrm{E}\left(\widehat{\operatorname{var}}\left(\widehat{\Delta}^{I}\right)\right)-\operatorname{var}\left(\widehat{\Delta}^{I}\right)$, the empirical relative root mean squared error RRMSE $=\left(\operatorname{MSE}\left(\widehat{\operatorname{var}}\left(\widehat{\Delta}^{I}\right)\right)\right)^{1 / 2} / \operatorname{var}\left(\widehat{\Delta}^{I}\right)$, and the coverage of the $95 \%$ confidence interval $\widehat{\Delta}^{I} \pm 1.96 \widehat{\operatorname{var}}\left(\widehat{\Delta}^{I}\right)^{1 / 2}$. The term $\operatorname{var}\left(\widehat{\Delta}^{I}\right)$ denotes the empirical variance computed from the 10000 observed values of $\widehat{\Delta}^{I}$. Computations were performed in $\mathrm{R}$ ( $\mathrm{R}$ Core Team, 2015) using some routines from the R packages 'sampling' (Tillé and Matei, 2013) and 'samplingVarEst' (Escobar and Barrios, 2014). We compare the proposed estimator $\widehat{V}\left(\widehat{\Delta}^{I}\right)$ from (25) versus a naïve approach which consists in treating the imputed values as real values. Note that there is no other competitor for the proposed approach, as design-based variance estimators for imputed change estimators is non existent in the literature.

Tables 1 and 2 give the RB, the RRMSE and the coverage for different values of the overlapping fraction $g$ between waves. In Table $1, \pi_{1 ; k}=n / N$ and in Table 2 the $\pi_{1 ; k}$ are proportional to the variable hours worked per week.

The proposed approach gives negligible RB. As expected, the naïve approach tends to severely underestimate the variance; in particular, when the fraction of non respondents is large; that is, when $q_{1 ; k}$ is small. Furthermore, by comparing Table 1 and 2, we observe smaller RB with unequal inclusion probabilities.

The proposed approach has smaller RRMSE than the naïve approach. However, with unequal probabilities we observe larger RRMSE. The coverage of the proposed approach is closer to $95 \%$. The coverage of the naïve approach is lower because of the under-estimation of the variance. 


\subsection{Missing not at random response and multiple imputation- classes}

Four variables, $y_{1}, y_{2}, x_{1}, x_{2}$ and $w_{1}$, are generated from a multivariate normal distribution with means $20,10,20,10$ and 20 . All the variables have the same variance equals to 5 . The correlation between $y_{1}$ and $y_{2}$ is either $\rho\left(y_{1}, y_{2}\right)=0.7$ or $\rho\left(y_{1}, y_{2}\right)=0.9$. The other correlations are $\rho\left(y_{\ell}, x_{\ell^{\prime}}\right)=\rho\left(y_{\ell}, w_{1}\right)=0.7$ and $\rho\left(x_{\ell}, x_{\ell^{\prime}}\right)=\rho\left(x_{\ell}, w_{1}\right)=0.5\left(\ell \neq \ell^{\prime}\right)$. The wave 1 variables are $y_{1}, x_{1}$ and $w_{1}$. The wave 2 variables are $y_{2}$ and $x_{2}$. We generate $N=20000$ values for each variables.

The values $y_{1 ; k}$ and $y_{2 ; k}$ are the values of the variable $y_{1}$ and $y_{2}$. The parameter of interest is the absolute change between means: $\Delta_{\mu}=\Delta / N$. The imputed estimator is $\widehat{\Delta}_{\mu}^{I}=\widehat{\Delta}^{I} / N$.

The sample $s_{1}$ is a randomised systematic sample with first-order inclusion probabilities $\pi_{1 ; k}$ proportional to $w_{1 ; k}$, where $w_{1 ; k}$ denotes the $k$-th value of $w_{1}$. The sample $s_{2}$ is a simple random sample of $n_{12}$ units selected from $s_{1}$ combined with a randomised systematic sample of $n_{2}-n_{12}$ units selected without replacement from $U \backslash s_{1}$ with probabilities proportional to $\pi_{1 ; k} /\left(1-\pi_{1 ; k}\right)$. We have that $\pi_{2 ; k} \bumpeq \pi_{1 ; k}$ (Berger and Priam, 2016). The sample sizes are $n_{1}=n_{2}=500$ and $n_{12}=375$. We consider that we have a single stratum. 10000 samples $s_{1}$ and $s_{2}$ are selected. The Hansen and Hurwitz (1943) variance estimator is used for cross-sectional variance estimation.

We consider hot-deck imputation with multiple imputation-classes as described in Section 7. The number of imputation classes is the same on wave 1 and 2: $C_{1}=$ $C_{2}=C$. We consider three types of imputation classes.

(i) "Population imputation classes": The imputation classes of wave $\ell$ are $C$ quantile classes based on the variable $x_{\ell}$. The bounds of the classes are the $(100 c / C) \%$ quantiles $(c=1, \ldots, C)$ of the population values $\left\{x_{\ell ; k}: k \in U\right\}$, where $x_{\ell ; k}$ de- 
notes the $k$-th value of $x_{\ell}$.

(ii) "Sample imputation classes": The imputation classes of wave $\ell$ are are $C$ quantile classes based on the sample values of the variable $x_{\ell}$. The bounds of the classes are the $(100 c / C) \%$ quantiles $(c=1, \ldots, C)$ of the sample values $\left\{x_{\ell ; k}: k \in s_{\ell}\right\}$.

(iii) "Across-waves imputation classes": For the classes of wave 1, we use $C$ quantile classes based on the sample values of the variable $x_{1}$, as in (ii). The wave 2 imputation classes are $C$ quantile classes based on the sample values $\left\{\widetilde{y}_{1 ; k}: k \in\right.$ $\left.s_{2}\right\}$, where

$$
\widetilde{y}_{1 ; k}= \begin{cases}y_{1 ; k}^{I} & \text { for } k \in s_{12}, \\ \widehat{\beta}_{0}+\widehat{\beta}_{1} x_{2 ; k} & \text { for } k \in s_{2} \backslash s_{1} .\end{cases}
$$

Here, $x_{2 ; k}$ is the value of the variable $x_{2}$ for unit $k$. The quantity $\widehat{\beta}_{0}$ and $\widehat{\beta}_{1}$ are the ordinary least square coefficients of the regression $y_{1 ; k}^{I}=\beta_{0}+\beta_{1} x_{2 ; k}$, with $k \in s_{12}$.

For the classes (i), the class indicators $b_{\ell ; k}^{(c)}$ do not depend on the samples selected. For the classes (ii) and (iii), the class indicators $b_{\ell ; k}^{(c)}$ depend on the samples. Note that the classes of wave 1 are different from the classes of wave 2 , unless $C=1$.

We consider a "missing not at random response mechanism". The first and second wave response probabilities $q_{1 ; k}$ and $q_{2 ; k}$ are given by $q_{\ell ; k}=\exp \left(\eta_{\ell ; k}\right)\left\{1+\exp \left(\eta_{\ell ; k}\right)\right\}^{-1}$, where $\eta_{1 ; k}=4-0.15 y_{1 ; k}$ and $\eta_{2 ; k}=3-0.2 y_{2 ; k}$. The resulting response probabilities lies within the range $[0.25,0.95]$. We have $a_{\ell ; k}=1$ if $u_{\ell ; k} \leq q_{\ell ; k}$ and $a_{\ell ; k}=0$ otherwise, where $u_{\ell ; k}$ are independent uniform random variables $U(0,1)$. The resulting response mechanism is missing not at random because large $q_{1 ; k}$ and $q_{2 ; k}$ are associated with 
small values of $y_{1 ; k}$ and $y_{2 ; k}$. The overall response rate are $73 \%$ and $72 \%$ for the first and second wave. The correlation between $q_{1 ; k}$ and $q_{2 ; k}$ is approximately 0.7 . The response probabilities are not constant within the imputation classes. Missing values are generated randomly before each selection of $s_{1}$ and $s_{2}$.

Table 3: Overall expectation, variance, root-mean squared error (RMSE) and coverage of $95 \%$ confidence interval based on the estimator proposed. Missing not at random response mechanisms. $\rho\left(y_{1}, y_{2}\right)$ denotes the correlation between the variables of interest. $N=20000, n_{1}=n_{2}=500$ and $n_{12}=375 . \Delta_{\mu}=\Delta / N$ and $\widehat{\Delta}_{\mu}^{I}=\widehat{\Delta}^{I} / N$.

\begin{tabular}{|c|c|c|c|c|c|c|c|c|}
\hline$\rho\left(y_{1}, y_{2}\right)$ & Imputation & $C$ & $\Delta_{\mu}$ & $E\left(\widehat{\Delta}_{\mu}^{I}\right)$ & $V\left(\widehat{\Delta}_{\mu}^{I}\right)$ & $E\left\{\widehat{V}\left(\widehat{\Delta}_{\mu}^{I}\right)\right\}$ & RMSE & Coverage (\%) \\
\hline \multirow[t]{10}{*}{0.7} & (i) Population & 1 & -10.03 & -10.09 & 0.022 & 0.021 & 0.0016 & 92.9 \\
\hline & level & 5 & -10.03 & -10.06 & 0.017 & 0.019 & 0.0029 & 95.9 \\
\hline & & 10 & -10.03 & -10.06 & 0.016 & 0.019 & 0.0031 & 96.0 \\
\hline & & 20 & -10.03 & -10.06 & 0.016 & 0.019 & 0.0036 & 96.3 \\
\hline & (ii) Sample & 5 & -10.03 & -10.06 & 0.016 & 0.019 & 0.0031 & 96.2 \\
\hline & level & 10 & -10.03 & -10.06 & 0.015 & 0.019 & 0.0037 & 96.7 \\
\hline & & 20 & -10.03 & -10.06 & 0.015 & 0.019 & 0.0039 & 96.8 \\
\hline & (iii) Across & 5 & -10.03 & -10.09 & 0.017 & 0.019 & 0.0029 & 94.5 \\
\hline & waves & 10 & -10.03 & -10.08 & 0.016 & 0.019 & 0.0033 & 95.3 \\
\hline & & 20 & -10.03 & -10.08 & 0.016 & 0.019 & 0.0032 & 95.2 \\
\hline \multirow[t]{10}{*}{0.9} & (i) Population & 1 & $\begin{array}{l}-9.99 \\
\end{array}$ & -10.06 & 0.019 & 0.019 & 0.0015 & 91.8 \\
\hline & level & 5 & -9.99 & -10.03 & 0.014 & 0.017 & 0.0030 & 95.7 \\
\hline & & 10 & -9.99 & -10.03 & 0.014 & 0.016 & 0.0031 & 96.0 \\
\hline & & 20 & -9.99 & -10.03 & 0.013 & 0.016 & 0.0031 & 96.2 \\
\hline & (ii) Sample & 5 & -9.99 & -10.03 & 0.013 & 0.017 & 0.0034 & 96.3 \\
\hline & level & 10 & -9.99 & -10.03 & 0.013 & 0.016 & 0.0035 & 96.2 \\
\hline & & 20 & -9.99 & -10.03 & 0.013 & 0.016 & 0.0033 & 96.3 \\
\hline & (iii) Across & 5 & $\begin{array}{l}-9.99 \\
\end{array}$ & -10.01 & 0.013 & 0.016 & 0.0032 & 96.8 \\
\hline & waves & 10 & -9.99 & -10.00 & 0.013 & 0.016 & 0.0037 & 97.3 \\
\hline & & 20 & -9.99 & -10.00 & 0.013 & 0.016 & 0.0035 & 97.2 \\
\hline
\end{tabular}

The simulation results are given in Table 3. Large number of classes reduces the bias of the point estimator. With a single imputation class $(C=1)$, the variance estimator has the smallest bias and is more stable (small root mean square error, 
RMSE), but with low coverages (92.9\% and 91.8\%). The low coverages is explained by the largest bias of the point estimator. Note that the point estimator is more precise with $C \geqslant 1$ and $\rho\left(y_{1}, y_{2}\right)=0.9$, in term of bias and variance. However, there is only negligible differences between the variance for $C \geqslant 5$. We only notice a decrease in the variance, as $C$ increases, for population level imputation classes with $\rho\left(y_{1}, y_{2}\right)=0.9$. For $C \geqslant 5$, we observe a slight positive bias for the variance estimator and an increase in the RMSE. For population level classes, the RMSE increases with $C$. The coverage observed are slightly larger than $95 \%$ for $C \geqslant 5$. We do not observe significant differences between the imputation classes (i), (ii) and (iii).

The missing not at random response mechanism tends to under-represent the large values of the variables of interest and therefore the observed correlation between $y_{1 ; k}$

and $y_{2 ; k}$ is lower than $\rho\left(y_{1}, y_{2}\right)$. As a result, the correlation between $\widehat{\tau}_{2}^{I}$ and $\widehat{\tau}_{1}^{I}$ is slightly under-estimated. This explains the slight positive bias for the variance estimator (Berger, 2004, p. 462). However, this bias is negligible because the coverages of the confidence intervals are of an acceptable order. This bias is only observed for $C \neq 1$. For $C=1$, the larger variance compensates the bias.

\section{Discussion}

The proposed variance estimator is applicable for unequal rotating stratified sampling designs when random hot-deck imputation is used at both waves and the sampling fractions are negligible. The proposed variance estimator may be extended in various ways. Point estimators, such as calibration estimators (Huang and Fuller, 1978; Deville and Särndal, 1992) which employ auxiliary population information may often be expressible as functions of totals. The proposed variance estimator (20) can be modified to accommodate this situation. 
The main advantages of the proposed variance estimator are that it is approximately unbiased under the response mechanisms and that it does not require the estimation of the response probabilities.

The proposed approach is not limited to hot-deck imputation, as it can be extended to other method of imputation, as long as the expectation of the imputed estimator of change under random imputation can be expressed as a function of totals.

It is possible to take into account of the wave to wave correlation by using a deterministic regression imputation technique. For example, we could impute by the fitted values of a regression model with the variable (34) as covariate. In that situation, the gradient (17) have a different expression and the term $B$ (see expression (12)) equals zero. The variance estimator can still be used. However, it does not take into account of the randomness of $\widehat{\beta}_{0}$ and $\widehat{\beta}_{1}$ in (34).

The variance estimator is based on the assumption that the imputation class are fixed. However, this assumption does not hold when the imputation classes are based on sampled data. This is also the case when the imputation at wave 2 is based on classes constructed from sample variables observed at wave 1. In Section 8.2, we suggest using the the wave 1 variable to impute at wave 2 , by using imputation classes based on the variable of interest of wave 1 (see (iii) "Across-waves imputation classes"). Our simulation study showed that sample based imputation classes have a negligible effect on the variance estimates, even with across-waves imputation classes. Adjusting the variance estimator to accommodate this situation is beyond the scope of this paper. This is a topic which would need further investigation. 


\section{Acknowledgements}

Yves G. Berger was supported by the grant RES-000-22-3045 of the Economic and

Social Research Council (UK). Emilio L. Escobar was supported by the National Council for Science and Technology (Mexico). We thank the reviewers for helpful comments and suggestions.

\section{References}

Andersson, C., Andersson, K., and Lundquist, P. (2011a), Estimation of change in a rotation panel design,, in Proc. 58th World Statist. Cong., Int. Statist. Inst., Dublin.

Andersson, C., Andersson, K., and Lundquist, P. (2011b), "Variansskattningar avseende förändringsskattningar i panelundersökningar (Variance Estimation of change in panel surveys)," Methodology reports from Statistics Sweden (Statistiska centralbyrån), .

Andridge, R. R., and Little, R. J. A. (2010), "A review of Hot Deck imputation for survey non-response," Int. Statist. Rev., 78, 40-64.

Berger, Y. G. (2004), "Variance estimation for measures of change in probability sampling," Canadian Journal of Statistics, 32(4), 451-467.

Berger, Y. G., and Priam, R. (2010), "Estimation of correlations between crosssectional Estimates from Repeated Surveys - an Application to the Variance of Change," Proceeding of the 2010 Symposium of Statistics Canada, .

Berger, Y. G., and Priam, R. (2016), "A Simple Variance Estimator of Change for Rotating Repeated Surveys: an Application to the EU-SILC Household Surveys," To appear in the Journal of the Royal Statistical Society, Series A, p. 22pp. 
Brick, J. M., and Montaquila, J. M. (2009), "Nonresponse and weighting," in Sample Surveys: Design, Methods and Applications, eds. D. Pfeffermann, and C. R. Rao, Vol. 29A of Handbook of Statistics, Amsterdam: Elsevier, pp. 163-185.

Deville, J. C., and Särndal, C. E. (1992), "Calibration Estimators in Survey Sampling," J. Amer. Statist. Assoc., 87(418), 376-382.

Deville, J. C., and Särndal, C. E. (1994), "Variance estimation for the regression imputed Horvitz-Thompson estimator," J. Off. Statist., 10, 381-394.

Escobar, E. L., and Barrios, E. (2014), samplingVarEst: Sampling Variance Estimation. $\mathrm{R}$ package version 0.9-9.

URL: http://cran.r-project.org/web/packages/samplingVarEst

Eurostat (2012), "European Union Statistics on Income and Living Conditions (EUSILC),", http://epp.eurostat.ec.europa.eu/portal/page/portal/microdata/eu_ silc.

Fay, B. E. (1991), "A design-based perspective on missing data variance," Proceeding of the 1191 Annual Research Conference. U.S. Bureau of the Census, pp. 429-440.

Fay, B. E. (1994), Analyzing imputed survey datasets with model-assisted estimators,, in Proc. Survey Methods Sec., Am. Statist. Assoc., pp. 900-905.

Gambino, J. G., and Silva, P. L. N. (2009), "Sampling and estimation in household surveys," in Sample Surveys: Design, Methods \& Applications, eds. D. Pfeffermann, and C. R. Rao, Vol. 29A of Handbook of Statistics, Amsterdam: Elsevier, pp. 407439.

Goga, C., Deville, J. C., and Ruiz-Gazen, A. (2009), "Use of functionals in lin- 
earization and composite estimation with application to two-sample survey data," Biometrika, 96, 691-709.

Hansen, M. H., and Hurwitz, W. N. (1943), "On the Theory of Sampling from Finite Populations," The Annals of Mathematical Statistics, 14(4), pp. 333-362.

Haziza, D. (2009), "Imputation and inference in the presence of missing data," in Sample Surveys: Design, Methods \& Applications, eds. D. Pfeffermann, and C. R. Rao, Vol. 29A of Handbook of Statistics, Amsterdam: Elsevier, pp. 215-246.

Haziza, D., and Beaumont, J. F. (2007), "On the Construction of Imputation Classes in Surveys," International Statistical Review, 75(1), 25-43.

Holmes, D. J., and Skinner, C. J. (2000), Variance Estimation for Labour Force Survey Estimates of Level and Change, London, England: Technical report, Government Statistical Service Methodology Series, 21.

Horvitz, D. G., and Thompson, D. J. (1952), "A generalization of sampling without replacement from a finite universe," J. Am. Statist. Assoc., 47, 663-685.

Huang, E. T., and Fuller, W. A. (1978), Nonnegative regression estimation for survey data,, in Proc. Soc. Statist. Sec., American Statistical Association, pp. 300-305.

Kalton, G. (2009), "Design for surveys over time," in Sample Surveys: Design, Methods 8 Applications, eds. D. Pfeffermann, and C. R. Rao, Vol. 29A of Handbook of Statistics, Amsterdam: Elsevier, pp. 89-108.

Kish, L. (1965), Survey Sampling, New York: Wiley.

Knottnerus, P., and van Delden, A. (2012), "On variances of changes estimated from rotating panels and dynamic strata," Survey Methodology, 38(1), 43-52. 
Lohr, S. L. (2009), Sampling: Design and Analysis, Boston: Brooks/Cole.

Münnich, R., and Zins, S. (2011), Variance Estimation for Indicators of Poverty and Social Exclusion,, Research Project Report WP3 - D3.2, FP7-SSH-2007-217322 AMELI.

URL: http://ameli.surveystatistics.net

Narain, R. (1951), "On Sampling without Replacement with Varying Probabilities," J. Ind. Soc. Agri. Statist., 3(3), 169-174.

Nordberg, L. (2000), "On variance estimation for measures of change when samples are coordinated by the use of permanent random numbers," Journal of Official Statistics, 16, 363-378.

Ohlsson, E. (1995), "Coordination of samples using permanent random numbers," In Business Survey Methods, eds. B. Cox, D.A. Binder, B.N. Chinnappa, A. Christianson, M. Colledge, and P.S. Kott, New York: Wiley and Sons, .

Qualité, L., and Tillé, Y. (2008), "Variance estimation of changes in repeated surveys and its application to the Swiss survey of value added," Surv. Methodol., 34, 173-181.

R Core Team (2015), R: A Language and Environment for Statistical Computing, R Foundation for Statistical Computing, Vienna, Austria. ISBN 3-900051-07-0.

URL: http://www.R-project.org/

Rao, J. N. K. (1965), "On two simple schemes of unequal probability sampling without replacement," Journal of the Indian Statistical Association, 3, 173-180.

Rao, J. N. K., and Shao, A. J. (1992), "Jackknife variance estimation with survey data under hotdeck imputation," Biometrika, 79, 811-822. 
Rao, J. N. K., and Sitter, R. R. (1995), "Variance estimation under two-phase sampling with application to imputation for missing data," Biometrika, 82, 453-460.

Robinson, P. M., and Särndal, C. E. (1983), "Asymptotic properties of the generalized regression estimator in probability sampling," Sankhya: The Indian Journal of Statistics, Series B, 43, 240-248.

Rubin, D. B. (1987), Multiple Imputation for Nonresponse in Surveys, New York: Wiley.

Sampford, M. R. (1967), "On Sampling Without Replacement with Unequal Probabilities of Selection," Biometrika, 54(3/4), 499-513.

Särndal, C. E., and Lundström (2005), Estimation in Surveys with Nonresponse, Chichester: Wiley.

Särndal, C. E., Swensson, B., and Wretman, J. (1992), Model Assisted Survey Sampling, New York: Springer.

Shao, J., and Steel, P. (1999), "Variance Estimation for Survey Data with composite Imputation and Nonnegligible Sampling Fractions," Journal of the American Statistical Association, 94, 254-265.

Smith, P., Pont, M., and Jones, T. (2003), "Developments in business Surv. Methodol. in the Office for National Statistics, 1994-2000," J. R. Statist. Soc. D (The Statistician), 52, 257-295.

Steel, P., and Fay, B. E. (1995), Variance estimation for finite populations with imputed data,, in Proceedings of the Survey Research Methods Section, American Statistical Association, pp. 374-379.

Tam, S. M. (1984), "On covariances from overlapping samples," American Statistician, 38(4), 288-289. 
Tillé, Y., and Matei, A. (2013), sampling: Survey Sampling. R package version 2.6. URL: http://cran.r-project.org/web/packages/sampling

Valliant, R., Dorfman, A. H., and Royall, R. M. (2000), Finite Population Sampling and Inference: A Prediction Approach, New York: Wiley.

Wolter, K. M. (2007), Introduction to Variance Estimation, 2nd edn, New York: Springer.

Wood, J. (2008), "On the Covariance Between Related Horvitz-Thompson Estimators," Journal of Official Statistics, 24(1), 53-78.

Yves G. Berger

Southampton Statistical Sciences Research Institute, University of Southampton, Highfield Campus, SO17 1BJ, United Kingdom

E-mail: y.g.berger@soton.ac.uk

Emilio López Escobar

Numérika, Av. Colonia del Valle 511 Piso 1, Col. Del Valle, 03100 D. F., México.

E-mail: emilio@numerika.mx 